\title{
Regenerative all-optical wavelength multicast for next generation WDM network and system applications
}

\author{
N. Yan - E. Tangdiongga $\cdot$ H. D. Jung $\cdot$ \\ I. Tafur Monroy • H. de Waardt . \\ A. M. J. Koonen
}

Received: 15 May 2007 / Accepted: 19 July 2007 / Published online: 14 September 2007

(C) Springer Science+Business Media, LLC 2007

\begin{abstract}
We experimentally demonstrate regenerative all-optical wavelength multicast by simultaneous multi-wavelength conversion of $10 \mathrm{~Gb} / \mathrm{s}$ non-return-to-zero signals to four ITU $100 \mathrm{GHz}$ spaced channels with a receiver sensitivity improvement of $1.84 \mathrm{~dB}$ and less than $0.14 \mathrm{~dB}$ difference among all the multicast channels, using a single commercial monolithically integrated SOA-MZI. The multicast device also exhibited about $22 \mathrm{~dB}$ optical signal-to-noise ratio enhancement for all the converted channels compared to the original signal channel without wavelength conversion. Our experiment for the first time revealed the regeneration properties of a SOA-MZI device for WDM wavelength multicast purposes, and proved the excellent performance of a simple scheme for various future network and system applications, such as all-optical wavelength routing and grid networking.
\end{abstract}

Keywords All-optical $\cdot$ Multicast $\cdot$ Multi-wavelength conversion $\cdot$ Regeneration $\cdot$ SOA-MZI $\cdot$ WDM

\section{Introduction}

Telecommunications networks are currently undergoing major transformations driven by new all-optical technologies

\footnotetext{
N. Yan $(\bowtie)$

Electrical Engineering, Eindhoven University of Technology, Den Dolech 2, PT 11.06, P.O. Box 513, Eindhoven 5600 MB, The Netherlands

e-mail: n.yan@tue.nl

E. Tangdiongga $\cdot$ H. D. Jung $\cdot$ H. de Waardt - A. M. J. Koonen Eindhoven University of Technology, Den Dolech 2,

Eindhoven $5600 \mathrm{MB}$, The Netherlands

I. Tafur Monroy

COM.DTU, Department of Communications, Optics and Materials,

Technical University of Denmark, Lyngby, Denmark
}

for wavelength division multiplexing (WDM) and wavelength routing applications. All-optical wavelength conversion reduces the blocking probability of the WDM network nodes, increases their transparency, and enables their dynamic wavelength assignments and allocation capability [1]. As wavelength conversion technologies are walking into a mature stage of high bit rates at $10-40 \mathrm{~Gb} / \mathrm{s}$ and successful research demonstrations at $160-320 \mathrm{~Gb} / \mathrm{s}$ [2] crossing the last two decades, all-optical multi-wavelength conversion (MWC) began to attract increasing interest in the last 5 years. Not only a huge amount of optic-electronic-optic (OEO) transponders and electronics can be saved for the same function, new applications are also emerging from this possibility. In particular, optical layer WDM wavelength multicast [3-6] and Grid networking [7] are becoming both technically feasible and commercially applicable.

Various all-optical MWC approaches have been reported. The mostly investigated MWC methods use the following techniques: four-wave mixing (FWM) [8,9], cross-phase modulation (XPM) [3-5], cross-gain modulation (XGM) [6, 7], cross-absorption modulation (XAM) [10], and fast nonlinear polarization switching (NPS) [11]. Among these, XPM multi-wavelength converters (MWCRs) based on a semiconductor optical amplifier (SOA)-Mach-Zehnder interferometer (MZI) excel the others by offering a great combination of advantages: satisfactory and leveled conversion efficiency across wide conversion bandwidth featured by the SOA gain spectra, simultaneous conversion of a considerable number of channels, wavelength flexibility, high operation speed, supporting both RZ and NRZ data format, power economical, commercial product availability, compactness, and integration potential. Moreover, SOA-MZIs have a broad area of applications in optical communications, which allows massive production to reduce the cost and increase the integrability. Its working principle and schematic are simple and 
straightforward, requiring no more complexity than any of the other methods reported. Finally, SOA-MZIs can support high data rates, such as $40 \mathrm{~Gb} / \mathrm{s}$, and potentially even higher bit rates in a push-pull configuration with fast SOAs.

In many occasions, SOA-MZIs have also demonstrated considerable signal regenerative effect [12], but so far this has never been reported when performing MWC. In this article, we demonstrate for the first time, to our knowledge, the regenerative simultaneous one-to-four MWC of $10 \mathrm{~Gb} / \mathrm{s}$ NRZ signals by a commercial SOA-MZI with negative power penalty of $1.84 \mathrm{~dB}$ and channel divergence of $0.14 \mathrm{~dB}$ at biterror rate (BER) of $10^{-9}$. All converted channels achieved optical signal-to-noise ratio (OSNR) enhancement of around $22 \mathrm{~dB}$ and satisfactory MWC efficiency of around $-3.11 \mathrm{~dB}$. Compared to the previously reported experiments [3,4], our results proved that SOA-MZIs can provide excellent performance for wavelength multicast applications, without the use of additional high-power assist light, as reported in [3] or push-pull configuration in [4] for simple $10 \mathrm{~Gb} / \mathrm{s}$ operation, and considerable signal regeneration can be obtained simultaneously, which are very essential and desirable features for future WDM network nodes in either opaque or all-optical infrastructure.

\section{Experimental setup and operation principle}

Figure 1 shows the experimental setup built with commercially available components, where all-optical wavelength multicast via a SOA-MZI was achieved by launching a data signal and several continuous waves (CWs) on the desired wavelength channels to the interferometric ports of the device. The monolithically integrated SOA-MZI wavelength converter was manufactured by Heinrich Hertz Institut (HHI) for $10 \mathrm{~Gb} / \mathrm{s}$ operation. ITU $100 \mathrm{GHz}$ grid wavelengths were deployed. A $10 \mathrm{~Gb} / \mathrm{s} \mathrm{NRZ} \mathrm{data} \mathrm{signal} \mathrm{was} \mathrm{generated} \mathrm{by} \mathrm{exter-}$ nally modulating a tunable $\mathrm{CW}$ laser source tuned to $1541.35 \mathrm{~nm}$, with pseudorandom bit sequence of pattern length $2^{31}-1$. Four CWs from 1544.53 to $1546.92 \mathrm{~nm}$ were combined by a four-to-one coupler and launched in the copropagating direction with the data signal. The average input power to $\mathrm{P} 1$ was kept at around $-1.13 \mathrm{dBm}$ and the total input power to $\mathrm{P} 2$ was around $0 \mathrm{dBm}$ with each individual $\mathrm{CW}$ channel alone adjusted to about $-6 \mathrm{dBm}$ at the output of the coupler. Simultaneously wavelength converted channels could be obtained from both arms P4 and P5, but only signals at $\mathrm{P} 4$ were selected for the BER test. The temperature of the SOA-MZI was kept at $20^{\circ} \mathrm{C}$ while SOA1 and SOA2 were biased at $242.96 \mathrm{~mA}$ and $310.95 \mathrm{~mA}$. All the BER measurements, including the back-to-back one, were performed keeping constant power of $0 \mathrm{dBm}$ to the photo diode, with the same default eye detection threshold level.

The SOA-MZI was operated in the standard configuration where the data signal was sent to only one of the two arms P1 to induce a phase shift on the CWs injected into P2 via XPM. The MZI translated the phase modulation into an amplitude modulation. As the phase change was only weakly dependent on the wavelength, single data streams could be simultaneously transferred onto multiple wavelength channels.

\section{Results and discussions}

Figure 2 presents the spectrum at the MWCR output P4. Data were converted onto all the four channels. The output OSNR for the converted channels was within the range of 38-43 dB. The SOA-MZI wavelength converter showed good conversion efficiency, because of the gain provided by the SOAs and the fact that the SOA-MZI converts phase modulation into amplitude modulation. The spectrum revealed a $4 \mathrm{~dB}$ average peak power ratio of the individual converted channels to the signal channel after the SOA-MZI MWC. If we define the conversion efficiency for a multi-wavelength converter as the average peak power ratio of the converted channels to the back-to-back signal channel bypassing the wavelength converter, the SOA-MZI exhibited a conversion efficiency of $-3.11 \mathrm{~dB}$ for the four simultaneously converted channels.

From Fig. 2, several FWM contributions due to the SOA nonlinear effect can be seen aside the input channels. The out-of-band FWM satellite signals were about at least $20 \mathrm{~dB}$ weaker than the desired wavelength channels. Therefore, it was expected that the in-band FWM products would be stronger. However, these FWM contributions did not degrade the BER performance of any of the simultaneously converted

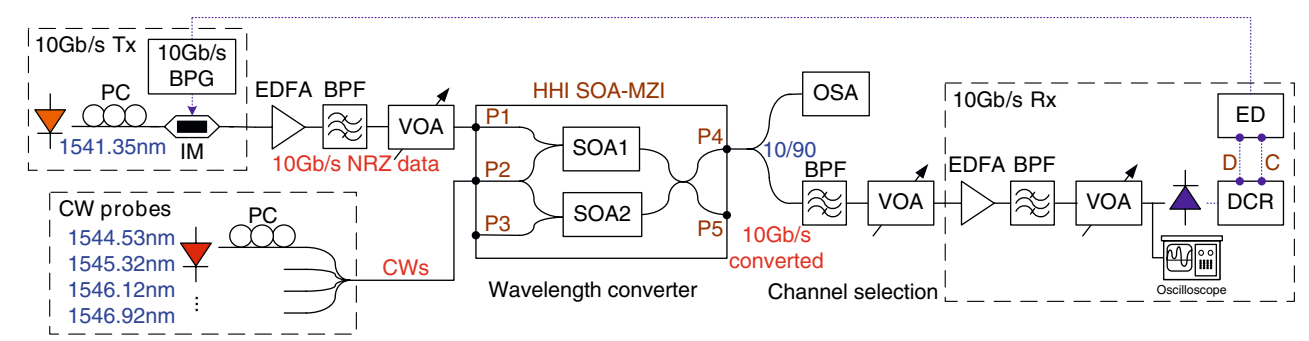

Fig. 1 Experimental setup for all-optical one-to-four wavelength multicast via a SOA-MZI. (Tx/Rx: Transmitter/Receiver; PC: polarization controller; BPG: bit-pattern generator; IM: intensity modulator; EDFA: erbium-doped fiber amplifier; BPF: bandpass filter; VOA: variable optical attenuator; OSA: optical spectrum analyzer; DCR: digital clock recovery; and ED: error detector) 


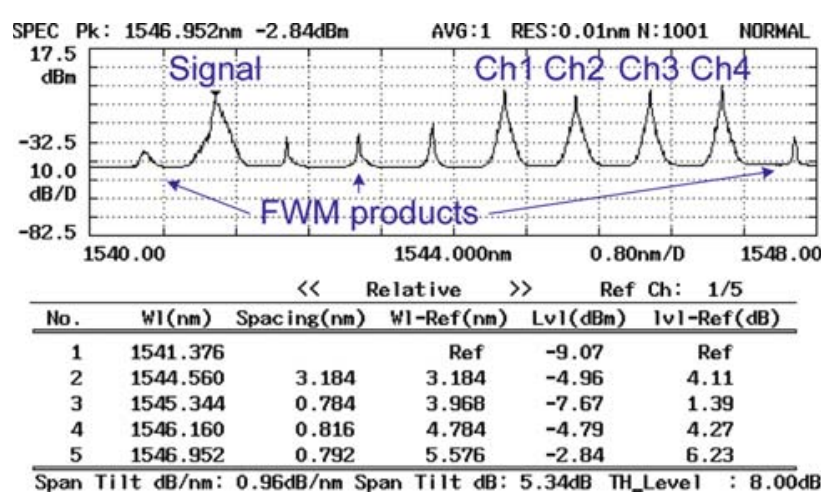

Fig. 2 Output spectrum at SOA-MZI port P4

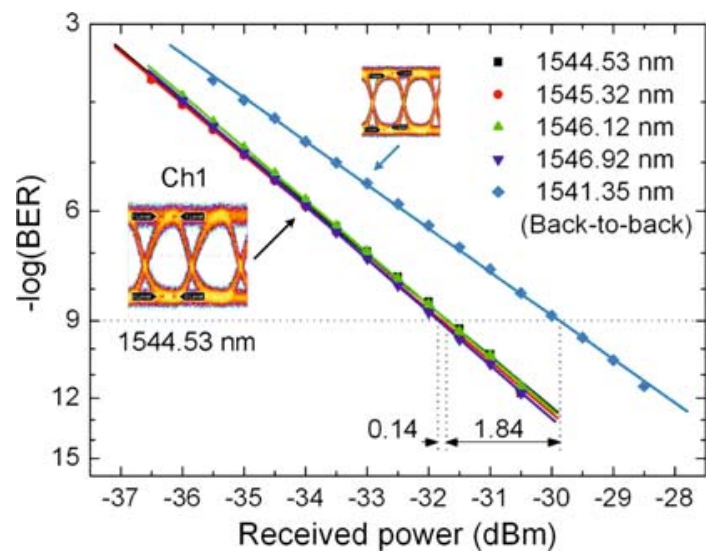

Fig. 3 BER results for all simultaneously converted channels vs. backto-back signal

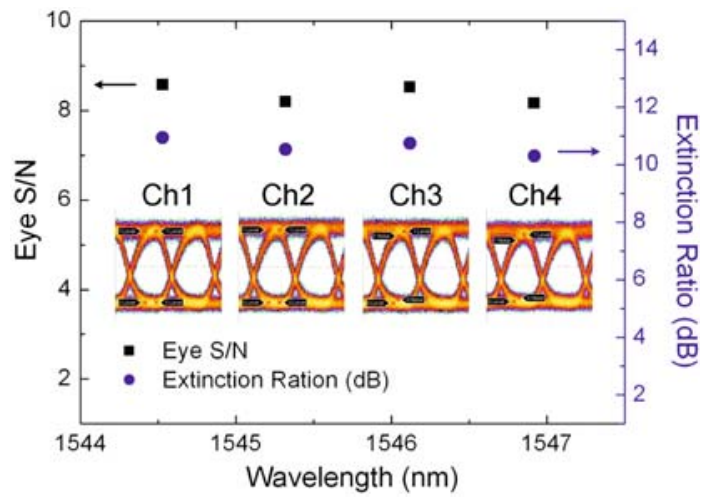

Fig. 4 Output eye signal-to-noise ratio (S/N)-left axis and extinction ratio (ER)-right axis of the four converted channels measured by oscilloscope with eye diagrams snapshots shown as insets

data signals, as shown in the following Fig. 3. Nor is the effect of FWM noticeable in the converted eye diagrams, which are presented in Fig. 4.

At the SOA-MZI output port P4, a BPF of $0.3 \mathrm{~nm}$ narrow bandwidth was used for the channel selection. At the receiver, after the preamplifier EDFA and the second BPF employed to remove the out-of-band ASE, the OSNRs of the four converted channels were around $41-45 \mathrm{~dB}$, with on average $22 \mathrm{~dB}$ enhancement over the back-to-back signal OSNR without wavelength conversion. The peak power ratio of the neighboring channels that were not completely suppressed by the filters to the channel under investigation was about $-33 \mathrm{~dB}$. By improving the OSNR of the original signals, the SOA-MZI MWCR demonstrated its signal regeneration capability. This feature is particularly desirable for WDM network nodes as in practice, optical cross connects inside a network often do not receive clean input signals directly from the source, but degraded optical data channels, due to the transmission loss and dispersion they have experienced crossing long distances. Optical signal regeneration capabilities are thus essential for the intermediate WDM nodes to improve the quality of the incoming data so that they can reach their final destination and be correctly detected.

In Fig. 3, BER measurements of all the four converted channels are plotted, with respect to the back-to-back channel. The eye diagrams of the back-to-back and one of the converted signals are shown as insets. The BER values indicated that the input data signal was converted to all the four channels with a receiver sensitivity improvement of $1.84 \mathrm{~dB}$ or more, while the sensitivity divergence among the four converted channels was measured to be no more than $0.14 \mathrm{~dB}$. The observed negative power penalty confirmed that the SOA-MZI increased the original signal quality by deeply modulating the signal phase and then translating the phase modulation into an amplitude modulation, which significantly cut down the noise level and possibly improved the rising and fall time of the signal eyes. This regeneration was probably also related to the fact that the four $\mathrm{CW}$ channels were copropagating with the data signal at the same time inside the SOA-MZI acting as internal assist lights for the channel under investigation. The BER results confirmed once again the signal regeneration properties of the simultaneous WDM wavelength multicast operation.

Finally, Fig. 4 demonstrates the output eye signal-to-noise ratios $(\mathrm{S} / \mathrm{N})$ and extinction ratios (ER) of the four simultaneously converted channels including their eye diagram snapshots, measured by an oscilloscope with $40-\mathrm{GHz}$ bandwidth limit. All the channels showed clear and widely open eyes with an average eye S/N of 8.36 and average ER of $10.63 \mathrm{~dB}$. The converted eye diagrams indicated a slightly lowered eye crossing point, which did not seem to have any negative influence on the system performance according to the BER results obtained. The clean multicast channels depicted by the eye snapshots further proved the excellent performance of the MWCR for applications in WDM wavelength-routed networks.

\section{Conclusions}

In order to propose SOA-MZI as an excellent candidate for future all-optical WDM wavelength multicast network nodes, 
we experimentally demonstrated that all-optical MWC based on a single commercial SOA-MZI can provide simultaneous regenerative conversion at $10 \mathrm{~Gb} / \mathrm{s}$, which to our knowledge has never been reported before. Previous demonstrations using SOA-MZIs for multicast at $10 \mathrm{~Gb} / \mathrm{s}$ have employed either an additional high optical power assist light [3] or a push-pull configuration that requires delicate fine tuning at the MZI input [4]. No noticeable signal regeneration has been observed in their results. While in our experiment, by deploying the simplest MWC scheme, we achieved $4 \times 10$ $\mathrm{Gb} / \mathrm{s}$ NRZ conversion of $100 \mathrm{GHz}$ spaced channels all with negative power penalty between $1.84-1.98 \mathrm{~dB}$ at BER of $10^{-9}$ and improved OSNR of around $22 \mathrm{~dB}$. The SOA-MZI wavelength converter also exhibited a satisfactory conversion efficiency of about $-3.11 \mathrm{~dB}$ for all the four channels. Our results confirmed the great potential of a SOA-MZI for next generation WDM network nodes with all-optical infrastructure, where optical layer wavelength multicast is a most desirable feature for the increasing volume of multimedia on-demand data services. Moreover, the signal regeneration properties of the SOA-MZI device we demonstrated are also essential for any intermediate optical cross connects that implement all-optical multicast technologies to be able to better the degraded incoming signal quality to allow for further long-distance propagation in the optical layer without the necessity of OEO conversion and electronic regeneration.

The number of MWC copies we demonstrated was restricted by our available laser sources. In our experiment, FWM byproducts observed from the output spectrum did not seem to be a limiting factor for the number of simultaneously converted channels, nor for the deployed channel spacing. Considering this promising performance, we expect that our apparatus can accommodate a much higher number of channels by adding additional $\mathrm{CW}$ probes, providing that they are located inside the SOA gain bandwidth. SOA-MZI MWC can also be applied for RZ signals [3,4]. Employing an MZI consisting of faster SOAs or deploying a push-pull scheme, the device can also be used for MWC at higher bit rate of $40 \mathrm{~Gb} / \mathrm{s}$ or more. Based on our results, we believe that this method can be suitable for various applications in next generation transparent all-optical WDM routing and networking.

Acknowledgements The authors would like to thank the European Commission for funding these research activities executed in the ISTLASAGNE project.

\section{References}

[1] Yoo, S.J.B.: Wavelength conversion technologies for WDM network applications. IEEE J. Lightwave Technol. 14(6), 955-966 (1996)

[2] Liu, Y., Tangdiongga, E., Li, Z., de Waardt, H., Koonen, A.M.J., Khoe, G.D., Dorren, H.J.S.: Error-free $320 \mathrm{~Gb} / \mathrm{s}$ SOA-based wavelength conversion using optical filtering. In: Proceedings of
Optical Fiber Communication Conference (OFC 2006), Anaheim, CA, USA, March 2006, PDP28

[3] Chung, H.S., Inohara, R., Nishimura, K., Usami, M.: All-optical multi-wavelength conversion of $10 \mathrm{Gbit} / \mathrm{s}$ NRZ/RZ signals based on SOA-MZI for WDM multicasting. Electron. Lett. 41(7): 432433 (2005)

[4] Reading-Picopoulos, D., Wang, F., Chai, Y.J., Penty, R.V., White, I.H.: $10 \mathrm{~Gb} / \mathrm{s}$ and $40 \mathrm{~Gb} / \mathrm{s}$ WDM multi-casting using a hybrid integrated Mach-Zehnder interferometer. In: Proceedings of Optical Fiber Communication Conference (OFC 2006), Anaheim, CA, USA, March 2006, OFP2

[5] Pleumeekers, J.L., Leuthold, J., Kauer, M., Bernasconi, P.G., Burrus, C.A., Cappuzzo, M., Chen, E., Gomez, L., Laskowski, E.: All-optical wavelength conversion and broadcasting to eight separate channels by a single semiconducter optical amplifier delay interferometer. In: Proceedings of Optical Fiber Communication Conference, pp. 596-597, (OFC 2002), March 2002, ThDD4

[6] Contestabile, G., Calabretta, N., Proietti, R., Ciaramella, E.: Double-stage cross-gain modulation in SOAs: an effective technique for WDM multicasting. IEEE Photonic Technol. Lett. 18(1): 181183 (2006)

[7] Lee, B.H.L., Mohamad, R., Dimyati, K.: Performance of all-optical multicasting via dual-stage XGM in SOA for grid networking. IEEE Photonic Technol. Lett. 18(21): 2215-2217 (2006)

[8] Contestabile, G., Presi, M., Ciaramella, E.: Multiple wavelength conversion for WDM multicasting by FWM in an SOA. IEEE Photonic Technol. Lett. 16(7): 1775-1777 (2005)

[9] Yan, N., Teixeira, A., Silveira, T., Tosi Beleffi, G.M., Curti, F., Forin, D., Dalla Longa, F., Tafur Monroy, I., Monteiro, P., Koonen, T.: Theoretical and experimental performance evaluation of all-optical multi-wavelength conversion by four-wave mixing in fiber at 10/20/40 Gb/s for optical layer multicast. In: Wiley Microwave Optic. Technol. Lett. 49(5): 1067-1071 (2005)

[10] Xu, L., Chi, N., Yvind, K., Christiansen, L.J., Oxenløwe, L.K., Mørk, J., Jeppesen, P., Hanberg, J.: $8 \times 40 \mathrm{~Gb} / \mathrm{s}$ RZ all-optical broadcasting utilizing an electroabsorption modulator. In: Proceedings of Optical Fiber Communication Conference (OFC 2004), March 2004, MF71

[11] Contestabile, G., Calabretta, N., Presi, M., Ciaramella, E.: Single and multicast wavelength conversion at $40 \mathrm{~Gb} / \mathrm{s}$ by means of fast nonlinear polarization switching in an SOA. IEEE Photonic Technol. Lett. 17(12): 2652-2654 (2005)

[12] Zhao, M., Morthier, G., Baets, R.: Demonstration of extinction ratio improvement from 2 to $9 \mathrm{~dB}$ and intensity noise reduction with the MZI-GCSOA all-optical 2R regenerator. IEEE Photonic Technol. Lett. 14(7): 992-994 (2002)

\section{Author Biographies}

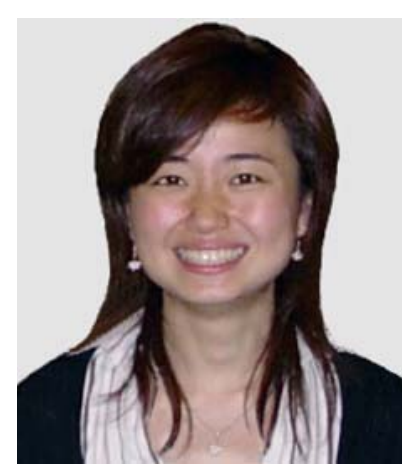

Ni Yan was born in Wuhan, China, in January 1979. She received her B.Eng./B.Sc. in Communication Engineering from Zhongshan University in Guangzhou, China, in June 2001. She worked as a Project Implementation Engineer at Siemens Transmission Systems Co. Ltd., the headquarters of Siemens Information \& Communications Networks in China between June 2001 and September 2002. She received her M.Sc. (Distinction) in Telecommunications (for 
industry) from University College London in September 2003 in London, UK. Since January 2004, she has been working as a PhD researcher in the Electro-Optical Communication Systems group at Eindhoven University of Technology, the Netherlands. She was involved in the European Commission funded projects IST-LASAGNE and ISTSTOLAS. Her current research interests mainly include optical labelswitched node architectures, all-optical multiwavelength conversion technologies and their applications, optical packet routers, as well as computer networks and information security.

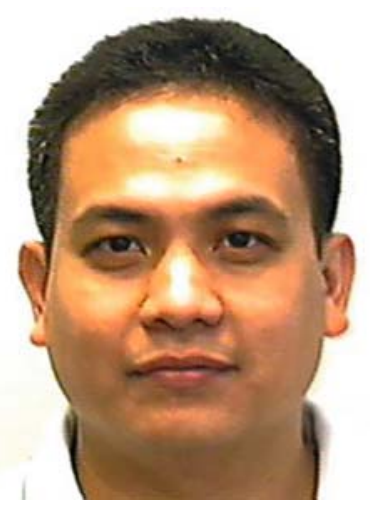

Eduward Tangdiongga is an Assistant Professor of the Eindhoven University of Technology TU/e, The Netherlands. He graduated in 1994 with the MSc degree and in 2001 the $\mathrm{PhD}$ degree, both from TU/e. In 1994 he joined the COBRA Research Institute where he conducted experimental works on the topic of crosstalk performance of multi-wavelength optical crossconnects. The work was carried out in two EU projects: BLISS (Broadband LIghtwave Sources and Systems) and APEX (Advanced Photonics Experimental X-connect). From 2001 till 2003 he participated in the EU project FASHION (ultraFAst Switching in HIgh-speed Optical time-division multiplexed Networks) for realization of $160 \mathrm{Gbit} / \mathrm{s}$ all-optical add-drop multiplexing and electro-optical $40 \mathrm{GHz}$ sub-harmonic clock recovery. He is currently working on all-optical wavelength conversion and demultiplexing for data rates of $\mathrm{Tb} / \mathrm{s}$ per wavelength. In 2005 he was on a sabbatical leave to Fujitsu Research Labs in Japan working on the topic of signal processing using highly nonlinear fibres and quantum-dot semiconductor optical amplifiers.

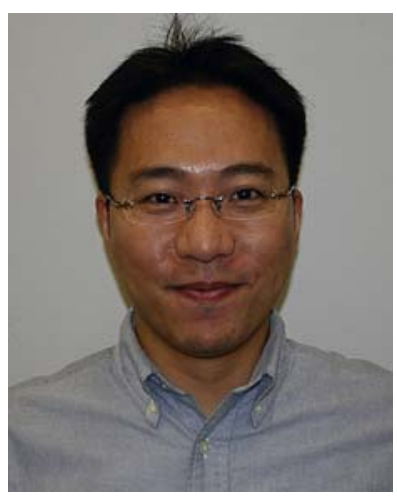

Hyun-Do Jung received the B.S. degrees in radio sciences and engineering from Kyunghee University, Korea, in 1999, and the $\mathrm{Ph} . \mathrm{D}$. degree in electrical and electronic engineering from Yonsei University, Korea, in 2005. Since Sep. 2005, he has been with the department of electrical engineering, Technical University of Eindhoven, the Netherlands as a postdoctoral researcher where he is involved in research on optical packet switching. His current research interests include optical systems for communications, optical packet switching, and microwave photonics technologies.

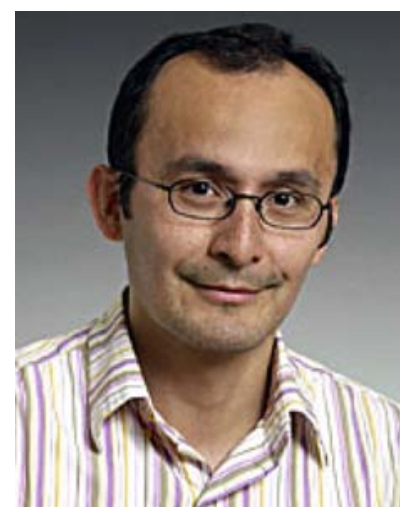

Idelfonso Tafur Monroy graduated from the Bonch-Bruevitch Institute of Communications, St. Petersburg, Russia, in 1992, where he received a M.Sc. degree in multichannel telecommunications. In 1996 he received a Technology Licenciate degree in telecommunications theory from the Royal Institute of Technology, Stockholm, Sweden. The same year he joined the Electro-Optical Communication Systems group of Eindhoven University of Technology, The Netherlands, where he earned a Ph.D. degree in 1999 and worked as an assistant professor until 2006. Currently he is an associate professor at the Department of Communications, Optics \& Materials (COM•DTU) at the Technical University of Denmark (DTU). He has participated in several European research projects (APEX, STOLAS, LASAGNE, MUFINS). His research interests are in optical label and packet switching, nanophotonic technologies and systems for integrated metro and access networks, and communication theory.

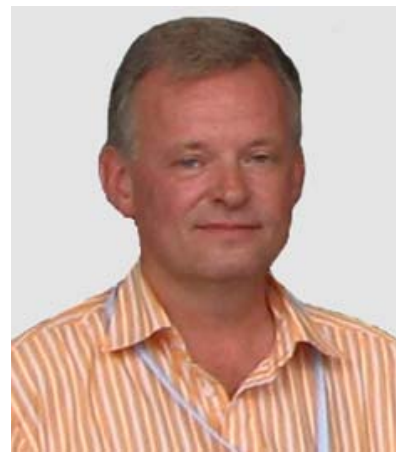

Huug de Waardt was born in Voorburg, The Netherlands, in December, 1953. He received the M.Sc.E.E. and the Ph.D. degrees from the Delft University of Technology, The Netherlands in 1980 and 1995, respectively. In 1981, he started his professional carrier in the Physics Department at PTT Research in Leidschendam, The Netherlands, where he worked on the performance issues of opto-electronic devices. In 1989

he moved to the Transmission Department and became involved in WDM high-bit-rate optical transmission. In 1995 he was appointed as an Associated Professor at the Eindhoven University of Technology (TU/e), Eindhoven, The Netherlands in the area of high-capacity trunk transmission. He coordinated the participation of TU/e in ACTS Upgrade, ACTS BLISS, ACTS APEX and IST FASHION. Presently he serves as project leader of the national research initiative Freeband Broadband Photonics (2004-2008). His current interests are in high capacity optical transmission and networking, integrated optics and semiconductor optical amplifiers/modulators. He (co)authored over 150 conference and journal papers. Dr. de Waardt is member of the IEEE. 


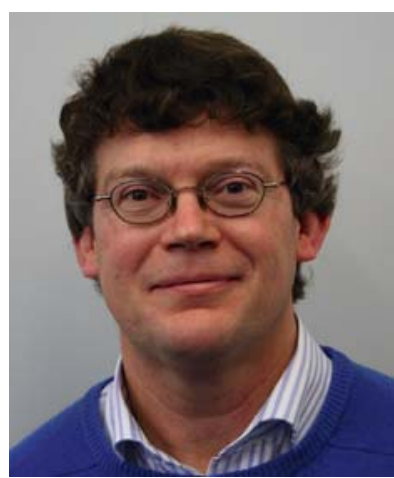

Antonius (Ton) M.J. Koonen (F'07-SM'01-M'00) received his M.Sc. (cum laude) in Electrical Engineering from Eindhoven University of Technology in 1979. He spent more than 20 years at Bell Laboratories in Lucent Technologies as a technical manager of applied research. $\mathrm{He}$ also is a Bell Labs Fellow since 1998 (the first one in Europe). Next to his industrial position, he has been a part-time professor at Twente University from 1991 to 2000. Since 2001, he is a full professor at Eindhoven University of Technology in the Electro-Optical Communication Systems group, which is a partner in the COBRA Institute; since 2004, he is the chairman of this group. His main interests are currently in broadband fibre access networks and in optical packet-switched networks. He has initiated and led several European and national R\&D projects in this area, on label-controlled optical packet routed networks (the EC FP5 IST project STOLAS), dynamically reconfigurable hybrid fiber access networks (EC FP4 ACTS TOBASCO on fiber-coax, EC FP4 ACTS PRISMA on fiber-wireless, EC FP5 IST HARMONICS on packet-switched access), and short-range multimode (polymer) optical fiber networks. Presently, he is involved in a number of access/in-home projects in the Dutch Freeband programme, in the Dutch IOP Generieke Communicatie programme, and in the EC FP6 IST Broadband for All programme (MUSE, e-Photon/ONe+, POF-ALL). 